\title{
Solving Multi-Sensor Multi-Target Assignment Problem Based on Compositive Combat Efficiency and QPSO Algorithm
}

\author{
Gongguo $\mathrm{Xu}^{1, \mathrm{a}}$, Xiusheng Duan ${ }^{1, \mathrm{~b}}$, Wenhua $\mathrm{Hu}^{1, \mathrm{c}}$, Hailong Zhang ${ }^{2, \mathrm{~d}}$ \\ ${ }^{1}$ Department of Electronic and Optical Engineering, Ordnance Engineering College, Shijiazhuang, \\ 050003, China \\ ${ }^{2}$ China Xi'an Satellite Control Center, Xi'an, 710043, China \\ aemail: xugguo@yeah.net, bemail:sjzdxsh@163.com, ${ }^{\mathrm{a} e m a i l: ~ h w h g a q @ s i n a . c o m, ~}{ }^{\mathrm{d}} \mathrm{email}$ : \\ woishailong@sina.com
}

Keywords: Multi-Sensor Multi-Target Assignment; Quantum Particle Swarm Optimization; Air Defense System

\begin{abstract}
Aiming at the multi-sensor multi-target assignment (MSMTA) problem under complex air defense combat environment, a new MSMTA model is proposed with the compositive combat efficiency of the identification, tracking and positioning stage. And then, the quantum particle swarm optimization (QPSQ) algorithm is imported in order to solve the MSMTA problem. Finally, the experiments show that the new MSMTA model is effective and compare the performances between the QPSO and particle swarm optimization (PSO) algorithms.
\end{abstract}

\section{Introduction}

With the development of the modern warfare, air defense combat environment is becoming more and more diversified and complicated with characteristics of whole-airspace, all-weather, multi-sensor, and multi-target. Therefore, the role of sensor management is becoming more and more prominent, especially in the $\mathrm{C}^{3} \mathrm{I}$ air defense system. The multi-sensor multi-target assignment (MSMTA) problem is the key problem in sensor management. In order to achieve maximum combat effectiveness and meet the real-time requirement in the battlefield, that the sensor resources are allocated to multiple targets quickly and rationally has became the research focus in the modern air defense, combat and command system.

MSMTA problem is a classic NP-hard complete problem belonging to the military operation research, which actually is the nonlinear and multiple constraints integer programming problem. The main difficulty is to solve the complex problem accurately and rapidly. There are lots of traditional solving methods, such as programming theory ${ }^{[1]}$, covariance control theory ${ }^{[2]}$, information theory ${ }^{[3]}$, game theory ${ }^{[4]}$, etc. Each method has its strengths and applicable situations.

On the basis of the above researches, this paper analyses the influence of each combat stage performance to the compositive combat efficiency based on the actual process of the air defense operation, and a new STA model is proposed with the compositive combat efficiency of the identification, tracking and positioning stage. The proposed model is more close to the actual battlefield environment. And then, the quantum particle swarm optimization (QPSQ) algorithm is imported to solve the MSMTA problem. Finally, the experiments show that the new MSMTA model and QPSQ algorithm are effective.

\section{MSMTA Problem Objective Function}

The traditional multi-sensor multi -target assignment methods generally regard a single aim as the objective principle of distribution, such as the maximum probability of detection, the optimal accuracy of the positioning ${ }^{[2]}$ or the maximum information gain ${ }^{[4]}$. However, in the actual air defense combat operation, a single aim does not describe the operational process, which has its limitations. Besides, the task of the sensor management becomes more and more complex. In the air defense combat, sensor tasks include search, interception, identification, tracking, and positioning. 
The performance of different sensor in different stage is different. Therefore, combined with the practical battlefield environment, a new allocation model is built based on the multistage compositive combat efficiency including identification, tracking, positioning stage. Furthermore, computing methods of combat efficiency fusion are also proposed. The calculation method is proposed as follows:

$$
\text { Gain }=c_{1} \cdot \text { Reco }+c_{2} \cdot \text { Trac }+c_{3} \cdot \text { Loca. }
$$

where, the Gain is the compositive combat efficiency, the Reco is identification combat ability, the Trac is tracking combat ability, the Loca is positioning combat ability; $c_{1} c_{2} c_{3}$ are the weights, and $c_{1}+c_{2}+c_{3}=1, \quad c_{1} c_{2} c_{3} \in[0,1]$, which can change according to the actual environment.

\section{MSMTA Problem Model}

Assuming in an air defense operation, the target number is $n$ represented as $T_{1}, T_{2}, \ldots T_{n}$; the sensor number is $m$ represented as $S_{1}, S_{2}, \ldots S_{m}$, and each sensor tracking ability is $C_{1}, C_{2}, \ldots C_{m}$; therefore, the tracking ability of the system is $C=C_{1}+C_{2}+\ldots+C_{m}$. When a target is tracked by two sensors simultaneously, the concept of virtual sensors is introduced in, which constitutes the new sensor. At the same time, the number of the sensor should be $\left(m^{2}+m\right) / 2$; the compositive combat efficiency can be calculated by the formula(1) shown in the matrix $\operatorname{Gain}\left[n,\left(m^{2}+m\right) / 2\right]$ :

$$
\text { Gain }=\left(\begin{array}{cccc}
G_{11} & G_{12} & \ldots & G_{1\left(m^{2}+m\right) / 2} \\
\ldots & \ldots & G_{i j} & \cdots \cdots \\
G_{n 1} & G_{n 2} & \ldots & G_{n\left(m^{2}+m\right) / 2}
\end{array}\right)_{n \times\left(m^{2}+m\right) / 2}
$$

where, $G_{i j}$ is the compositive combat efficiency when the sensor $i$ is assigned to the target $j$. Furthermore, the assignment matrix is defined as $R=\left[r_{i j}\right]_{n \times m}$, when the sensor $i$ is assigned to the target $j, r_{i j}=1$; otherwise, $r_{i j}=0$ 。

Through the above analysis, the STA problem in air defense operation can be converted to the following model:

$$
\text { Maximize } \sum_{i=1}^{n} P\left(T_{i}\right) \cdot \operatorname{Gain}(i, R)
$$

and the constraint conditions are as follows:

$$
\left\{\begin{array}{c}
\sum_{j=1}^{m} r_{i j} \leq 2 \\
\sum_{i=1}^{n} r_{i j} \leq C_{j} \\
\sum_{j=1}^{m} \sum_{i=1}^{n} r_{i j} \leq C \\
t \leq T_{\max }
\end{array}\right.
$$

where, $C_{j}$ is the tracking ability of sensor $j, \quad C$ is the tracking ability of the system, $T_{\max }$ is time limit. $P\left(T_{j}\right)$ is target priority determination of sensor $j$.

\section{Quantum Behaved Particle Swarm Optimization Algorithm}

After the establishment of the MSMTA model, the problem has been transformed into a combinatorial optimization problem. Especially when the numbers of sensors and targets are large, the complexity of this problem is relatively high. In view of the traditional PSO algorithm easily falls into the local optimization and has slow convergence speed, the QPSO algorithm is imported to solve the problem in this paper.

In order to avoid disadvantages of the general particle swarm optimization (PSO) algorithm. On the basis of quantum theory, the quantum behaved particle swarm optimization algorithm is 
proposed by Sun Jun ${ }^{[5]}$. This method gives the quantum behaved particle, that is, the position of the particle is described by the wave function, and the particle state is decided by the Schrodinger Equation. The specific particle position equation is as following:

$$
x(t)=Q \pm L / 2 \ln (1 / \mu)
$$

where, the $\mu$ is the random number on the [0,1], $L 、 Q$ can be calculated by the formula(6) and formula(7).

$$
\begin{aligned}
& L(t+1)=2 \beta(t)|\operatorname{mbest}(t)-x(t)| \\
& Q(t)=\varphi p_{i b}(t)+(1-\varphi) p_{g b}(t)
\end{aligned}
$$

where, $\beta=1-0.5($ iter / iterm), iter is the iterations, iterm is the maximum number of iterations, mbest is the average value of particles, $\varphi$ is the random number on the $[0,1], p_{i b}(t)$ is the best value of particle $i, p_{g b}(t)$ is the best value of all particles. And then, the particle position equation is shown below.

$$
x(t)=\varphi p_{i b}(t)+(1-\varphi) p_{g b}(t)+\operatorname{rand}(t) \cdot 2 \beta(t)|\operatorname{mbest}(t)-x(t)| \ln (1 / \mu)
$$

where,

$$
\operatorname{rand}(t)=\left\{\begin{array}{cc}
1 & \text { if }(\psi \leq 0.5) \\
-1 & \text { if }(\psi \geq 0.5)
\end{array}\right.
$$

and $\psi$ is also the random number on the [0,1]。Compared with the general PSO algorithm, QPSO algorithm, because of the introduction of the characteristic of quantum particle, the whole searching ability of QPSO algorithm is improved greatly.

\section{Simulation Results}

Assuming in an air defense operation, the target number is 6 represented as $T_{1}, T_{2}, T_{3}, T_{4}, T_{5}, T_{6}$; the sensor number is also 6 represented as $S_{1}, S_{2}, S_{3}, S_{4}, S_{5}, S_{6}$, in which the tracking ability of $S_{5} 、 S_{6}$ is 3 and the tracking ability of $S_{1} 、 S_{2} 、 S_{3} 、 S_{4}$ is only 1; and the compositive combat efficiency is shown in Table 1; every target is allocated up to 2 sensors; the particle numbers is 150 and the maximum number of the iteration is 200.

Table 1 The total combat effectiveness of sensor-target assignment

\begin{tabular}{cllllll}
\hline & $T 1$ & $T 2$ & $T 3$ & $T 4$ & $T 5$ & $T 6$ \\
\hline S1 & 4.41 & 4.28 & 4.88 & 4.81 & 4.31 & 5.14 \\
S3 & 4.52 & 5.05 & 4.55 & 5.52 & 5.00 & 4.87 \\
S4 & 4.09 & 3.65 & 4.39 & 3.92 & 4.29 & 4.54 \\
S5 & 4.37 & 4.68 & 4.98 & 5.25 & 5.02 & 5.11 \\
S6 & 4.45 & 4.65 & 5.55 & 5.80 & 4.15 & 5.46 \\
S1 \&S2 & 5.46 & 4.72 & 4.43 & 4.10 & 4.26 & 5.87 \\
S1 \&S3 & 5.44 & 5.40 & 6.24 & 6.32 & 5.61 & 6.14 \\
S1 \&S4 & 5.25 & 5.12 & 6.61 & 6.39 & 5.25 & 5.62 \\
S1 \&S5 & 4.81 & 5.03 & 6.04 & 6.10 & 6.22 & 6.04 \\
S1 \&S6 & 5.04 & 5.32 & 6.21 & 6.49 & 4.88 & 6.58 \\
S2 \&S3 & 6.23 & 5.78 & 5.46 & 5.33 & 5.04 & 7.10 \\
S2 \&S4 & 4.89 & 5.53 & 5.84 & 5.90 & 5.02 & 5.34 \\
S2 \&S5 & 5.17 & 5.55 & 5.88 & 6.37 & 5.89 & 5.84 \\
S2 \&S6 & 5.10 & 5.57 & 6.34 & 6.77 & 5.68 & 6.11 \\
S3 \&S4 & 4.70 & 4.88 & 5.19 & 5.54 & 5.17 & 5.43 \\
S3 \&S5 & 4.63 & 4.77 & 6.18 & 6.34 & 4.97 & 5.76 \\
S3 \&S6 & 5.86 & 5.10 & 4.95 & 4.53 & 5.14 & 6.63 \\
S4 \&S5 & 5.41 & 5.70 & 6.64 & 6.92 & 6.46 & 6.26 \\
S4 \&S6 & 6.06 & 6.01 & 5.94 & 5.63 & 5.71 & 6.87 \\
S5 \&S6 & 5.96 & 5.50 & 6.42 & 6.47 & 4.78 & 6.85 \\
\hline
\end{tabular}


Fig 1 The optimal allocation scheme

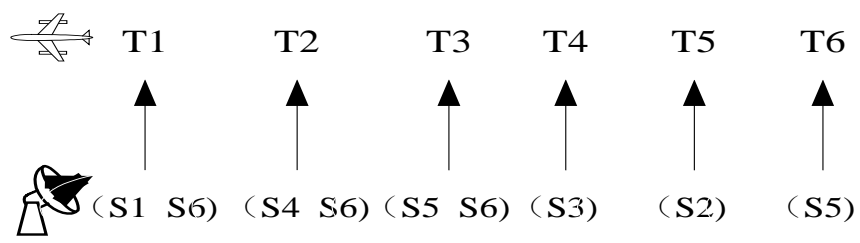

The optimal allocation scheme of the Simulation Results is shown in Fig 8. The best compositive combat efficiency is 14.8467 , and the consumption time is $0.47 \mathrm{~s}$, which meets the real-time requirement. Besides, the results are also meet other conditions. Every target is allocated up to 2 sensors. Every target allocated to one sensor is not more than the tracking ability of the sensor. In summary, the experiment shows that the improved algorithm is effective.

For the further analysis of the performance of QPSO algorithm, through the experiment, the QPSO and PSO algorithms were compared. Selecting the top 100 iteration steps in a process of optimization, the global best values are shown in Figure 2. From the figure, the QPSO algorithm has a better searching ability than the PSO algorithm and QPSO, which has better global optimization ability and consumes less time.

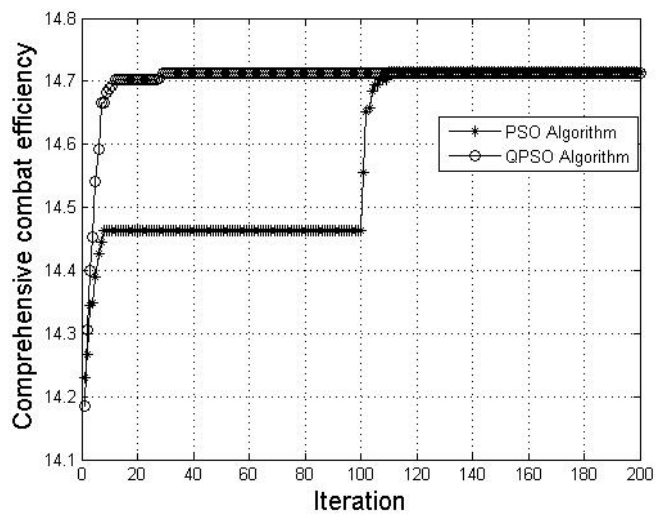

Fig 2 The comparison of convergence curves of the three algorithms

On the basis of the above experiments, repetitive operation of 200 times, the experimental results are shown in Table 2. Obviously, the average optimization value and the success rate through the QPSO algorithm are both better than the PSO algorithm. The variety of 200 experimental results are respectively shown in the Figure 3 and Figure 4, it is obvious to see, the QPSO algorithm searching is more stable and has better optimization results.

Table 2 The comparison of algorithm performances

\begin{tabular}{rll}
\hline & PSO & QPSO \\
\hline Average Optimization Value & 14.56 & 14.78 \\
Success Rat & 0.20 & 0.56 \\
Average Steps Consumed & 55.29 & 26.33 \\
\hline
\end{tabular}




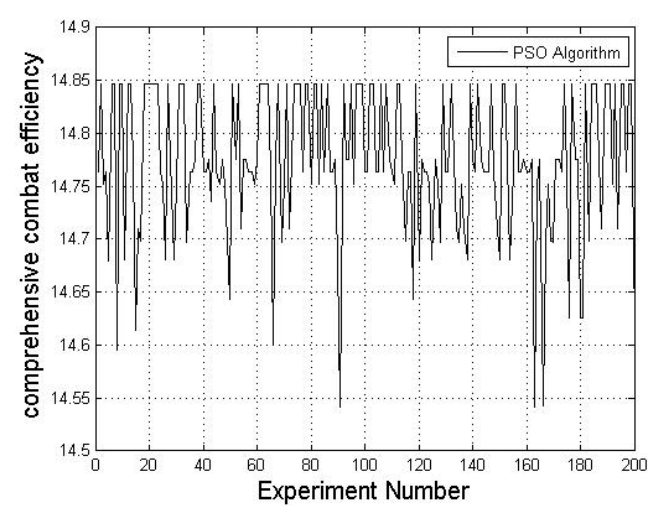

Fig 3 The optimization results of PSO algorithm

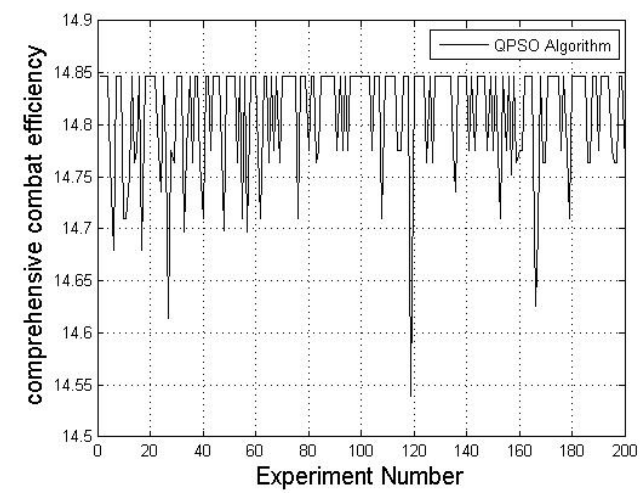

Fig 4 The optimization results of QPSO algorithm

\section{Conclusions}

This paper has focused on the problem of the multi-sensor multi-target assignment (MSMTA) under complex aerial defense combat environment. The study can effectively solve the MSMTA problem, and has a great effect to improve the ability of the ground air defense combat. Moreover, the QPSQ algorithm also has great application value for other multi-attribute decision making problems. In the future, the research effort should be focused on the improvement of the QPSQ algorithm.

\section{References}

[1] Chhetri A S, Morrell D, Papandreou- Suppappola A. Sensor resource allocation for tracking using outer approximation[J]. IEEE Signal Processing Letters, 2007, 14(3): 213-216.

[2] Zhou Wenhui, Hu Weidong, Yu Wenxian. An Adaptive Sensor Allocation Algorithm with Covariance Control[J]. Signal Processing, 2005, 21 (1):57-62.( In Chinese)

[3] Fowler M L, Chen M, Binghamton S. Fisher information based data compression for estimation using two sensors[J]. IEEE Transactions on Aerospace and Electronic Systems, 2005, 41(3):1131-1137.

[4] MA Fei, CAO Ze-yang, LIU Hui. Construction and search of strategy space of target assignment based on game theory[J]. Systems Engineering and Electronics, 2010, (9):1941-1945. (In Chinese)

[5] Sun J. A global search strategy of quantum-behaved particle swarm optimization[C]//Proceedings o f IEEE conference on Cybernetics and Intelligent Systems, 2004: 111- 116. 